\title{
Terapia manual em pacientes portadores de hérnia discal lombar: revisão sistemática
}

\author{
Manual therapy in patients with hernia discal lombar: \\ systematic review
}

Gabriella Maria Lima Verde Loiola

Ana Vanessa Araujo Pedrosa²

Brenna Barbosa da Silva ${ }^{2}$

Edinice de Sousa Modesto ${ }^{2}$

Thiago Brasileiro Vasconcelos ${ }^{3}$

Francisca Daiane de Oliveira Santos ${ }^{2}$

Vasco Pinheiro Diógenes Bastos ${ }^{4}$

\section{RESUMO}

A hérnia de disco é uma alteração que pode acometer qualquer parte da coluna vertebral, sendo mais frequente na região lombar. A hérnia de disco surge como resultado de diversos pequenos traumas na coluna que vão, com o passar do tempo, lesando as estruturas do disco intervertebral, ou pode acontecer como consequência de um trauma severo sobre a coluna. Esse trabalho objetivou analisar nas bases de dados as técnicas de Terapia Manual utilizadas em pacientes portadores de hérnia discal lombar. Tratou-se de uma revisão sistemática, transversal e com estratégia de análise quantitativa dos resultados, realizada no período de agosto a novembro de 2013. A busca foi realizada nas bases de dados Pubmed e Bireme (Medline, Lilacs, Pedro, Scielo) nos últimos 10 anos. Foram incluídos 12 artigos no período de 2001 a 2013 onde $66,4 \%(n=8)$ relacionaram a terapia manual eficaz, melhorando alguns parâmetros nos pacientes e 33,2\% $(n=4)$ não demonstraram resultados após alguns exercícios e alongamentos. Com este estudo pode-se concluir que o programa de terapia manual para pacientes portadores de hérnia de disco lombar proporcionou melhora no quadro álgico, na funcionalidade, na flexibilidade, no ganho de força e na mobilidade articular permitindo que os pacientes conseguissem voltar a realizar as atividades de vida diária.

\section{PALAVRAS-CHAVES:}

Lombalgia;Hérnia de disco; Fisioterapia;Terapia manual.

\footnotetext{
${ }^{1}$ Fisioterapeuta pela do Centro Universitário Estácio do Ceará.

${ }^{2}$ Discente do curso de Fisioterapia do Centro Universitário Estácio do Ceará.

${ }^{3}$ Fisioterapeuta. Mestre em Farmacologia pela Universidade Federal do Ceará.

${ }^{4}$ Fisioterapeuta. Doutor em Farmacologia. Docente do curso de Fisioterapia do Centro Universitário Estácio do Ceará.
} 


\section{ABSTRACT}

A herniated disc is a change that can affect any part of the spine, being most common in the lumbar region. A herniated disc comes as a result of several minortrauma in the column that will, over time, damaging the structures of the intervertebral disc, or it can happen as a result of severe trauma on the spine. This study aimed to analyze the databases techniques of manual therapy used in patients with lumbar disc herniation. This was a systematic review, transverse and strategy for the quantitative analysis of the results, carried out in the period August to November 2013. The search was performed in Pubmed and Bireme (Medline, Lilacs, Pedro,SciELO) in the last 10 years. We included 12 articles in the period from 2001 to 2013 where $66.4 \%$ (n $=8$ ) were related to manual therapy effective, improving some parameters in patients and $33.2 \%(n=4)$ showed no results after a few exercises and stretches. With this study we can conclude that the program of manual therapy for patients with lumbar disc herniation provided improvement in pain symptoms, Functionality, flexibility,gain strength and joint mobility allowing patients were able to return to perform the activities daily life.

\section{KEYWORDS:}

Lumbago, Herniated Disc, Physiotherapy,Pilates, Manual Therapy 


\section{INTRODUÇÃO}

A hérnia de disco é uma alteração que pode acometer qualquer parte da coluna vertebral, sendo mais frequente na região lombar. A composição do disco intervertebral é responsável pela hidratação do núcleo e pela distribuição das pressões uniformes sobre o anel. Com a diminuição dos componentes hídricos do disco, ocorre um aumento da pressão sobre as fibras anulares que se tornam suscetíveis a rupturas (1,2). $^{(1)}$

A coluna lombar, por ser uma região de grande mobilidade, sofre forças de compressão, tornando o núcleo pulposo vulnerável à deslocamentos. Com o passar do tempo, em consequência de um trauma severo sobre a coluna, lesa as estruturas do disco intervertebral, sendo geralmente mais comuns nos segmentos L4-L5, L5-S1. Alguns dos mecanismos que favorecem a degeneração com consequente projeção do núcleo são: desequilíbrios musculares, esforços nas atividades de vida diária (AVD's) e posturas que facilitam a desorganização da distribuição das pressões do disco ${ }^{(3)}$.

O diagnóstico deve ser basicamente clínico, complementado por exame de imagem para melhor acerto do nível envolvido ${ }^{(4,5)}$.

Com o passar do tempo, se o movimento não é restabelecido na articulação, os músculos ao redor dela começam a se encurtar, perdendo a flexibilidade. Com isso, o espaço entre uma vértebra e outra, destinado aos nervos, começa a diminuir, comprometendo o impulso nervoso. Além disso, o disco intervertebral diminui de espessura como resultado da diminuição do aporte de nutrientese oxigênio e pelo fato de que o peso não é mais absorvido e distribuído igualmente pela falta de movimento articular. ${ }^{(6)}$.

0 termo lombalgia na hérnia de disco se refere à dor na coluna lombar, sendo essa, uma disfunção que acomete ambos os sexos, podendo variar de uma dor súbita à dor intensa e prolongada, o padrão de recorrência da dor lombar é elevado $30 \%$ a $60 \%$ dos casos estão relacionados ao trabalho, a perda do movimento, ligada ao esforço excessivo ou repetitivo, maus hábitos posturais e disfunções biomecânicas que podem levar ao aparecimento da hérnia de disco, porque ao levantar um peso do chão com o tronco inclinado para frente, sem dobrar os joelhos, o disco sofre uma força na sua porção anterior, empurrando o conteúdo do núcleo pulposo para trás e forçando a ruptura do anel fibroso ${ }^{(7,8)}$.

As dores lombares, em especial, atingem níveis epidêmicos na população mundial. Estimativas mostram que cerca de 70 a $85 \%$ de toda a população mundial irá sentir dor lombar em alguma época de sua vida determinando elevados custos ao sistema de saúde e afetando vários segmentos sociais e econômicos ${ }^{(9,10)}$.

A dor lombar pode ser aguda, caracterizada por dor na última semana, nos indivíduos que relataram positividade para dor nos últimos 12 meses e crônica, quando há presença de dor por sete semanas ou mais ${ }^{(11,12)}$.

Cerca de 95\% das hérnias de disco lombar são devidas a compressões das raízes $\mathrm{L} 5$ e $\mathrm{S} 1$, sendo incomum, a herniação L3 e L4. São típicas em pacientes na faixa etária de 30 aos 60 anos e têm uma história natural bem conhecida: geralmente são recorrentes e as crises tendem a durar em média 2 a 3 meses. $\mathrm{Na}$ maior parte dos casos, são hérnias de bom prognóstico e raramente necessitam de tratamento cirúrgi$\mathrm{CO}^{(13)}$.

O tratamento conservador tem oferecido os meIhores resultados nos indivíduos com hérnia discal lombar, embora nem todos que tenham hérnia discal lombar consigam constatação nos exames de imagem. O processo de reabsorção do núcleo pulposo ainda não está totalmente esclarecido, merecendo futuras investigações sobre o assunto. A atividade física tem colaborado no tratamento da hérnia de disco lombar, mas ainda não estão esclarecidos, quais são especificamente os melhores exercícios para cada etapa da crise de dor $(14,15,16)$.

O método conservador da região lombar tem associação com diferentes metodologias auxiliares, como uso de cintos e coletes, a manipulação, o programa de atividade física, a tração, a crioterapia, a acupuntura e a prescrição de analgésicos e antiinflamatórios. O procedimento cirúrgico é outra opção disponível para o tratamento da hérnia de disco, embora sua indicação ocorra quando o curso natural do processo em questão segue uma piora significativa após o uso de medidas não agressivas. Poucos estu- 
dos existem comparando a eficácia entre os tratamentos conservador e cirúrgico ${ }^{(17)}$.

Quando se busca a analgesia através do relaxamento superficial pelo aumento da temperatura tecidual, a termoterapia infravermelha é bem empregada e usada quando um aumento na temperatura do tecido é o objetivo do tratamento. O benefício mais efetivo das modalidades infravermelhas é oferecer analgesia ou reduzir a sensação de dor associada à lesão. Em níveis mais profundos, o ultrassom também pode ser empregado como forma secundária de calor já que seu efeito é eliminar o quadro inflamatório. A crioterapia se enquadra na conduta de tratamento em que o objetivo principal é retirar o calor tecidual associado à analgesia ${ }^{(18,19)}$.

O tratamento da dor e disfunção lombar envolve uma equipe multidisciplinar, incluindo médico, fisioterapeuta, educador físico, enfermeiro e psicólogo, tendo como proposta geral, controlar o quadro álgico e a promoção do bem-estar e do retorno às atividades funcionais do indivíduo. A fisioterapia tem diversos recursos terapêuticos que auxiliam na promoção do alívio sintomático da dor e na reabilitação destes pacientes ${ }^{(20,21)}$.

Quanto aos tipos de intervenções podemos citar: realização de atividade física, orientações para exercícios, fisioterapia convencional, treinamento de força, resistência e coordenação, programa individual de treinamento físico, alinhamento postural, alongamento e fortalecimento, readequação ergonômica do ambiente de trabalho, aconselhamento ergonômico, em alguns casos são utilizados grupos terapêuticos atualmente, sendo essas intervenções, em alguns estudos, aplicadas isoladamente, e em outros havia a combinação de uma ou mais ${ }^{(22,23)}$.

A Terapia Manual é o principal recurso da Fisioterapia Manipulativa, que se trata de uma especialidade dentro da Fisioterapia no nível de pós-graduação, para a reabilitação de pacientes.A pompagem é um trabalho miotensivo com mobilização somada ao deslizamento das fáscias, que tem como objetivo o relaxamento muscular, melhora na nutrição circulatória dos tecidos moles e articulações, quebra de contraturas/encurtamentos/retrações e restauração do formato ou comprimento ${ }^{(24)}$.
Dentre os recursos utilizados está o método Pilates desenvolvido por JopephPilates no início da década de 1920 que tem como base um conceito denominado de contrologia, que é a correta consciente de todos os movimentos musculares do corpo, é a correta utilização e aplicação dos mais importantes princípios das forças que atuam em cada um dos ossos do esqueleto, com o completo conhecimento dos mecanismos funcionais do corpo, e o total entendimento dos princípios de equilíbrio e gravidade aplicados a cada movimento, no estado ativo, em repouso e dormindo ${ }^{(25)}$.

No uso da terapia manual está incluso também a Reeducação Postural Global (RPG) que é um método de alongamento muscular ativo, descrito originalmente em 1987, por Philippe Emmanuel Souchard, com o princípio de alongar em conjunto os músculos antigravitários, e foi baseado na compreensão das cadeias musculares posturais. Este método é amplamente difundido e tem sido muito utilizado como conduta fisioterapêutica em alterações posturais, principalmente nas desordens da coluna vertebral; porém, sua ação sobre o sistema respiratório é pouco documentada (26).

Com isso, este trabalho se justifica com um propósito de mostrar que existe um meio destes pacientes obterem uma melhora no seu quadro clínico através de um tratamento fisioterápico de hérnia discal lombar.

Sendo assim, esse estudo contribuiu de uma forma positiva, oferecendo aos profissionais da área de saúde perspectivas acerca de informações relacionadas à atuação da fisioterapia em pacientes portadores de hérnia discal lombar, relacionando com a terapia manual incluindo a Pompage, o RPG e o Pilates auxiliando na melhora do quadro clínico do paciente, com o intuito de mostrar a influência da fisioterapia no tratamento destes pacientes.

Esse trabalho tem por objetivo geral analisar nas bases de dados as técnicas de Terapia Manual utilizadas em pacientes portadores de hérnia discal lombar. E como objetivo específico caracterizar a hérnia discal de acordo com o quadro clínico relatado nas bases de dados e analisar as técnicas que podem ser utilizadas nesses pacientes com hérnia discal lombar. 


\section{METODOLOGIA}

O presente trabalho foi um estudo com estratégia quantitativa dos resultados apresentados. Que abrangeu a bibliografia já tornada pública em relação ao tema estudado, propiciando o exame de um tema sobre novo enfoque ou abordagem.

Foram utilizadas fontes tornadas públicas em relação ao tema estudado, tendo como forma de leitura crítica, seletiva, reflexiva e analítica. A consulta ocorreu no período de agosto a novembro de 2013, sendo usado como descritores: lombalgia, hérnia de disco, fisioterapia, pilates, terapia manual.

Sendo incluídos artigos relacionados à Terapia Manual bem como as suas propriedades e os diversos meios usados pelo fisioterapeuta no tratamento quanto à hérnia discal lombar. Os artigos utilizados foram os dos últimos 10 anos. Foram excluídos os artigos que citavam a palavra terapia manual, mas não discutiam sobre o tema, artigos incompletos, ou que não puderam ser visualizados e com idade superior a 10 anos.

Os artigos que foram selecionados seguiram as perspectivas da análise temática, sendo inicialmente procedida à leitura de todo acervo, a identificação dos eixos temáticos e aferidos seus respectivos núcleos de sentido para em seguida separar o material que foi utilizado no estudo, também foram submetidos à classificação do tipo de metodologia empregada em cada estudo. Os assuntos foram analisados e interpretados, sendo agrupados considerando semelhanças e diferenças das informações dos autores. O texto foi construído sendo registrado após leitura crítica-analítica com objetivo de selecionar a ideia principal de cada trabalho pesquisado.

\section{RESULTADOS}

Foram encontrados nas bases de dados durante esta pesquisa um total de 20 artigos, porém apenas
$60 \%(n=12)$ foram incluídos, pois estes vinham ao encontro do objetivo proposto neste trabalho.

No quadro 1, são apresentadas as características gerais dos estudos encontrados, onde $8,3 \%(n=1)$ foram publicados no ano de 2001, 8,3\% $(n=1)$ no ano de $2003,8,3 \%(n=1)$ no ano de $2004,24,9 \%$ $(n=3)$ no ano de $2005,16,6 \%(n=2)$ no ano de 2009, $8,3 \%(n=1)$ no ano de $2010,8,3 \%(n=1)$ no ano de 2011 e 16,6\% $(n=2)$ no ano de 2012.

Quanto ao país da pesquisa foram realizados nos Estados Unidos da América 24,9\% $(n=3)$ e 74,7\% $(n=9)$ foram realizados no Brasil (Quadro 1).

Diante dos objetivos expostos em cada manuscrito, 33,2\% $(n=4)$ tinham como objetivo, comparar os efeitos da terapia manual ealívioda dor,8,3\% $(n=1)$ demonstrar situações associadas a hérnia de disco, $24,9 \%(n=3)$ analisar os efeitos do método pilates, $16,6 \%(n=2)$ avaliar a atuação e a eficácia do RPG nas lombalgias, $8,3 \%(n=1)$ analisar os efeitos das técnicas de pompage na hérnia de disco e $8,3 \%(n=1)$ relacionar situações importantes associadas à lombalgia nos aspectos ergonômicos.(Quadro 1).

O tipo de estudo, 33,2\% $(n=4)$ são estudos bibliográficos, 8,3\% $(n=1)$ são estudos clínicos, fisiológicos e preventivos, $41,5 \%(n=5)$ são estudos de caso, $8,3 \%$ são estudos de literatura e $8,3 \%(n=1)$ são estudos seletivos (Quadro 1).As características dos artigos quanto a resultados e considerações finais podem ser observados no Quadro 2.

Quanto aos resultados encontrados, 66,6\% $(n=8)$ obtiveram melhora da dor nos parâmetros avaliados e 33,4\% $(n=4)$ não obtiveram melhora(Quadro2).

Diante das considerações finais expostas em cada trabalho podemos verificar que $66,4 \%(n=8)$ relacionaram a terapia manual eficaz, melhorando alguns parâmetros nos pacientes e 33,2\% $(n=4)$ não demonstraram resultados após alguns exercícios e alongamentos(Quadro2).

\begin{tabular}{|l|l|l|l}
\hline Autor & Ano & Resultados & $\mathrm{Cc}$ \\
\hline
\end{tabular}


Quadro 1 - Distribuição dos dados de acordo com as características gerais dos artigos encontrados. Fortaleza/CE, 2013

\begin{tabular}{|c|c|c|c|c|}
\hline Autor & Ano & País & Objetivos & Tipo de estudo \\
\hline Negrelli(27) & 2001 & Brasil & $\begin{array}{l}\text { Aliviar a dor, o aumento da capaci- } \\
\text { dade funcional e o retardamento } \\
\text { da progressão da doença. }\end{array}$ & Revisão Bibliográfica \\
\hline Santos ${ }^{(28)}$ & 2003 & EUA & $\begin{array}{l}\text { Demonstrar situaçoes associadas à } \\
\text { hérnia de disco. }\end{array}$ & $\begin{array}{l}\text { Uma revisão clínica, fi- } \\
\text { siológica e preventiva. }\end{array}$ \\
\hline Heineck $^{(29)}$ & 2004 & EUA & $\begin{array}{l}\text { Avaliar um programa de terapia } \\
\text { manual em relação à dor e à fun- } \\
\text { cionalidade do paciente portador } \\
\text { de hérnia de disco lombar }\end{array}$ & Estudo de caso \\
\hline Saccoet al. ${ }^{(30)}$ & 2005 & Brasil & $\begin{array}{l}\text { Analisar visão cinesiológica e bio- } \\
\text { mecânica do método Pilates e com- } \\
\text { pará-los para uma melhor descri- } \\
\text { ção do método e dos benefícios } \\
\text { desta atividade. }\end{array}$ & Estudo de caso \\
\hline $\begin{array}{l}\text { Basilio, } \\
\text { Parra }\end{array}$ & 2005 & Brasil & $\begin{array}{l}\text { Propor o pilates como tratamento } \\
\text { para hérnia de disco lombar. }\end{array}$ & Revisão Bibliográfica \\
\hline Macedo, Brigano(32) & 2009 & Brasil & $\begin{array}{l}\text { Comparar os efeitos da terapia ma- } \\
\text { nual e cinesioterapia na dor, quali- } \\
\text { dade de } \\
\text { vida e incapacidade de pacientes } \\
\text { com lombalgia }\end{array}$ & Estudo de caso \\
\hline Folhadela, Meija ${ }^{(33)}$ & 2009 & Brasil & $\begin{array}{l}\text { Avaliar a atuação, identificação dos } \\
\text { tipos, formas e a eficácia do RPG no } \\
\text { tratamento da lombalgia. }\end{array}$ & Revisão bibliográfica \\
\hline Briganó, Macedo ${ }^{(34)}$ & 2005 & Brasil & $\begin{array}{l}\text { comparar os efeitos da terapia ma- } \\
\text { nual em pacientes com lombalgia. }\end{array}$ & Estudo de caso \\
\hline Monteiro, Rangel ${ }^{(35)}$ & 2010 & Brasil & $\begin{array}{l}\text { Analisar o efeito das técnicas de } \\
\text { pompagem no alívio da dor em tra- } \\
\text { tamento de hérnia discal lombar }\end{array}$ & Estudo de caso \\
\hline Teodoriet al. ${ }^{(36)}$ & 2011 & Brasil & $\begin{array}{l}\text { Realizar os efeitos da intervenção } \\
\text { fisioterapêutica utilizando o méto- } \\
\text { do RPG. }\end{array}$ & Revisão de Literatura. \\
\hline $\begin{array}{l}\text { Tenório, } \\
\text { Vieira }^{(37)}\end{array}$ & 2012 & EUA & $\begin{array}{l}\text { Relacionar importantes situações } \\
\text { associadas à lombalgia, com ênfase } \\
\text { nos aspectos ergonôminos no tra- } \\
\text { balho e postura sentada. }\end{array}$ & Revisão Seletiva. \\
\hline $\begin{array}{l}\text { Costa, } \\
\text { Roth, } \\
\text { Noronha }{ }^{(38)}\end{array}$ & 2012 & Brasil & $\begin{array}{l}\text { Analisar os efeitos da utilização do } \\
\text { método pilates, por meio de uma } \\
\text { revisão da literatura com estudos } \\
\text { realizados no Brasil. }\end{array}$ & Revisão Bibliográfica \\
\hline
\end{tabular}


Quadro 2 - Distribuição dos dados de acordo com o ano, resultados e conclusão dos estudos analisados. Fortaleza/CE, 2013.

\begin{tabular}{|c|c|c|c|}
\hline Negrelli $^{(27)}$ & 2001 & $\begin{array}{l}\text { Os resultados obtidos com a aplicação } \\
\text { de algumas técnicas não têm demons- } \\
\text { trado efeitos positivos no alívio da dor. }\end{array}$ & $\begin{array}{l}\text { A terapia conservadora tem sido a prefe- } \\
\text { rida como a primeira escolha de trata- } \\
\text { mento para o alívio da dor. }\end{array}$ \\
\hline Santos ${ }^{(28)}$ & 2003 & $\begin{array}{l}\text { Tiveram um bom resultado na postura, } \\
\text { alongando-se e mantendo por } 20 \text { s. }\end{array}$ & $\begin{array}{l}\text { Medidas de correções posturais e suges- } \\
\text { tões de alongamento. }\end{array}$ \\
\hline Heineck $^{(29)}$ & 2004 & $\begin{array}{l}8 \text { pacientes tiveram melhora da dor na } \\
\text { posição deitada, } 7 \text { pacientes ainda sen- } \\
\text { tem dor durante à noite. }\end{array}$ & $\begin{array}{l}\text { Após umas } 10 \text { sessões de fisioterapia eles } \\
\text { obtiveram uma melhora no quadro. }\end{array}$ \\
\hline Saccoet al. ${ }^{(30)}$ & 2005 & $\begin{array}{l}\text { Diminuição da amplitude de movimen- } \\
\text { to. Não teve melhora da dor. }\end{array}$ & $\begin{array}{l}\text { A coluna lombar é exercitada intensa- } \\
\text { mente através de alongamentos e de al- } \\
\text { guns exercícios. }\end{array}$ \\
\hline $\begin{array}{l}\text { Basilio, } \\
\text { Parra }\end{array}$ & 2005 & $\begin{array}{l}\text { Melhora na tolerância ao exercício e na } \\
\text { qualidade de vida, melhora na autoes- } \\
\text { tima. }\end{array}$ & $\begin{array}{l}\text { Conclui com um programa de qualidade } \\
\text { de vida para melhorar esses pacientes } \\
\text { com hérnia de disco através do Pilates. }\end{array}$ \\
\hline Macedo, Brigano ${ }^{(32)}$ & 2009 & $\begin{array}{l}\text { Em recursos de terapia manual e cine- } \\
\text { sioterapia apresentou efeitos significa- } \\
\text { tivos na melhora da dor, qualidade de } \\
\text { vida e incapacidade dos pacientes com } \\
\text { lombalgia. }\end{array}$ & $\begin{array}{l}\text { A terapia manual e cinesioterapia são ne- } \\
\text { cessárias na reabilitação dos pacientes } \\
\text { com lombalgia e apresentam-se eficien- } \\
\text { tes no tratamento dador lombar. }\end{array}$ \\
\hline Folhadela, Meija ${ }^{(33)}$ & 2009 & $\begin{array}{l}\text { Verificou-se diferença entre os tipos e as } \\
\text { formas da eficácia do RPG. Teve melho- } \\
\text { ra. }\end{array}$ & $\begin{array}{l}\text { O programa melhorou a qualidade de } \\
\text { vida desses pacientes com hérnia discal } \\
\text { lombar. }\end{array}$ \\
\hline $\begin{array}{l}\text { Briganó, } \\
\text { Macedo (34) }\end{array}$ & 2005 & $\begin{array}{l}\text { Foram } 25 \text { pacientes, sendo que foi en- } \\
\text { contrada uma diferença significante } \\
\text { após o tratamento fisioterápico e tam- } \\
\text { bém na mobilidade da coluna lombar. } \\
\text { Houve uma melhora no quadro. }\end{array}$ & $\begin{array}{l}\text { Conclui que a terapia manual tem in- } \\
\text { fluencia significativa na melhora da lom- } \\
\text { balgia e da mobilidade da coluna lombar. }\end{array}$ \\
\hline $\begin{array}{l}\text { Monteiro, } \\
\text { Rangel (35) }\end{array}$ & 2010 & $\begin{array}{l}\text { Antes do tratamento, a paciente relata- } \\
\text { va dor, com o método de pompage hou- } \\
\text { ve alívio da dor. }\end{array}$ & $\begin{array}{l}\text { A aplicação da pompagem lombar em } \\
\text { caso de hérnia discal na mesma região } \\
\text { reduziu o quadro da dor. }\end{array}$ \\
\hline Teodoriet al. ${ }^{(36)}$ & 2011 & $\begin{array}{l}\text { Foram encontrados } 25 \text { estudos, sendo } \\
13 \text { relacionados ao método RPG e oito } \\
\text { aos alongamentos globais e ativos, e } \\
\text { sentiram melhoras em todos. }\end{array}$ & $\begin{array}{l}\text { Parte dos estudos aponta que o método } \\
\text { RPG é mais efetivo, enquanto outros } \\
\text { mostram resultados similares a outros } \\
\text { métodos de intervenção fisioterapeutica }\end{array}$ \\
\hline $\begin{array}{l}\text { Tenório, } \\
\text { Vieira }{ }^{(37)}\end{array}$ & 2012 & $\begin{array}{l}\text { Programa de exercícios e formas de pos- } \\
\text { turas e não tiveram melhora. }\end{array}$ & $\begin{array}{l}\text { Os resultados revelam que a postura in- } \\
\text { correta foi o que causa as dores lomba- } \\
\text { res. }\end{array}$ \\
\hline $\begin{array}{l}\text { Costa, } \\
\text { Roth, } \\
\text { Noronha }\end{array}$ & 2012 & $\begin{array}{l}\text { Através do pilates eles ganharam força, } \\
\text { melhora da dor e flexibilidade }\end{array}$ & $\begin{array}{l}\text { O método pilates melhora o fortaleci- } \\
\text { mento da musculatura, alémo método } \\
\text { pilates melhora o fortalecimento da } \\
\text { musculatura }\end{array}$ \\
\hline
\end{tabular}




\section{DISCUSSÃO}

No estudo feito por Negrelli ${ }^{(27)}$ mostra os princípios e benefícios de exercícios apropriados que são bem conhecidos e a motivação do paciente para executar atividade física que geralmente é maior durante duas a três semanas após o período de inabilidade. A hérnia de disco é uma frequente desordem músculo-esquelética, responsável pela lombociatalgia.

Alguns estudos, como, Santos ${ }^{(28)}$,Macedo, Brigano ${ }^{(32)}$ e Tenório, Vieira ${ }^{(37)}$, mostram situações importantes associadas à lombalgia com um programa de terapia manual em relação à dore a funcionalidade do paciente portador de hérnia de disco lombar.

Heineck ${ }^{(29)}$ demonstrou que, na análise tardia de uma amostra de 15 pacientes com hérnia discal lombar, oito pacientes sentem dor na posição deitada e sete pacientes apresentaram melhora a dor durante a noite, ao deitar, ao realizar atividade física leve, ao sair da cama pela manhã, ao pegar um objeto no chão e ao carregar sacolas pesadas.

No estudo feito por Saccoet al. ${ }^{(30)}$, Basilio, Parra $^{(31)}$ e Costa ${ }^{(28)}$ mostram uma visão cinesiológica e biomecânica do método Pilates caracterizando-se por um conjunto de movimentos onde a posição neutra da coluna vertebral é sempre respeitada, objetivando a melhora da cordenação da respiração com o movimento do corpo, flexibilidade geral e força muscular e postura.

Folhadela, Meija(33), Teodoriet al. ${ }^{(36)}$ demonstraram que os efeitos de intervenção fisioterapêutica através do método RPG indicaram benefícios do método na melhora da força muscular respiratória, expansibilidade torácica, mobilidade toracoabdominal e da pressão respiratória máxima, além de reduzir a dor.

No presente estudo de Briganó, Macedo ${ }^{(34)}$ e Monteiro,Rangel ${ }^{(35)}$ mostram que a terapia manual tem influencia significativa na melhora da lombalgia e da mobilidade da coluna lombar através de várias técnicas de pompage promovendo o relaxamento muscular melhorando o quadro álgico dos pacientes.

\section{CONCLUSÃO}

O programa de terapia manual para pacientes portadores de hérnia de disco lombar proporcionou me-
Ihora no quadro álgico, na funcionalidade, na flexibilidade, no ganho de força e na mobilidade articular permitindo que os pacientes conseguissem voltar a realizar as atividades de vida diária com qualidade demonstrando eficácia no tratamento aplicado.

Após a realização deste trabalho, conclui-se conforme os resultados obtidos, que as técnicas de terapia manual estão demonstrando resultados positivos aos pacientes portadores de hérnia de disco lombar, evitando assim, que muitos sejam submetidos a processo cirúrgico.

\section{REFERÊNCIAS}

1. BARROS, S.S. Lombalgia ocupacional e a postura sentada. Revista Dor, 2011; 12 (3): 226-230.

2. HELFENSTEIN, J.M.; GOLDENFUM, M.A. Siena, C. Lombalgia ocupacional. Revista da Associação Médica Brasileira, 2010; 56 (5): 583-589

3. ANDRADE, E.N.; PORTO, G.; SANTOS, G.M. O alívio da dor através da estimulação nervosa transcutânea acupuntural e burst em hérnia discal lombar L4-L5/ L5-S1 no estágio agudo. Fisioterapia Brasil, set./out.2004; 5 (5): 344-349

4. ORTIZ, J.; ABREU, A.D. Tratamento cirúrgico das hérnias discais lombares em regime ambulatorial. Revista Ortopédica Brasileira, nov./dez.2000; 35 (12): 440-446

5. GRACITELLI, M.E.C.; BARROS, F.; IUTAKA, A.S. Mapeamento do trajeto extraforaminal da raiz $L 4$ no espaço intertransversário L4-L5 através do acesso paramediano a coluna vertebral. Acta Ortopédica Brasileira, São Paulo, 2006; 14 (5): $30-35$

6. ANTÔNIO, S. F. Abordagem diagnóstica e terapêutica das dores lombares. Revista Brasileira Medicina, São Paulo, 2002; 59 (6): 449-461

7. MARRAS, W.S. Occupational low back disorder causation and control. Revista Ergonomics, London, 2000; 43 (7): 880-902

8. BERNARD, C. Lombalgia e lombociatalgias em medicina ocupacional. Revista Brasileira de Medicina, 1993;50(11): 3-9

9. MILANI, J.P.; MARTINS, M.R.I.; SILVA, E.C.; ROCHA, C.E. A qualidade de vida no período pré e pós- operatório de pacientes portadores de hérnia de disco lombar. Revista Dor, 2009; 10(1): 33-37

10. PITANGA, F.J.G.; LESSA, I. Prevalência e fatores associados ao sedentarismo no lazer em adultos. Caderno Saúde Pública, 2005;21(3): 870-877 
11. ANDERSSON, G.B. Epidemiological features of chronic low-back pain. Lancet, 1999; 354(9178): 581-585

12. ARKIE, A.; GUERRA, K. Hérnia de disco lombar: saiba mais, para evitar ou tratar. Revista Contra Relógio, Ed.162, Março 2007

13.CAILLIET, R. Lombalgias: síndromes dolorosas. São Paulo: Editora Manole: $1979 \cdot$ p.96- 111

14. WETLER, E, BARROS, J.F. O tratamento conservador através da atividade física na hérnia de disco lombar. Revista Digital, Buenos Aires, março 2004; 10 (70): 140-145

15. CECIN, H.A. Diagnóstico e tratamento das lombalgias e lombociatalgias. Projeto Diretrizes. Associação Médica Brasileira e Conselho Federal de Medicina. 2001.

16. DECCACHE, T.; SILVA, M.A.G. Método terapêutico-pedagógico no tratamento da dor lombar. Fisioterapia Brasil, 2007; 8(1): 36-40

17. NEGRELLI, W.F. Hérnia Discal: procedimentos de tratamentos. Acta Ortopédica Brasileira, São Paulo out./dez. 2001;9(4):39-45

18. GABRIEL, M.R.; PETIT, J.D.; CARRIL, M.L. Fisioterapia em Traumatologia, Ortopedia e Reumatologia. $1^{a}$ Ed.São Paulo, 2001.

19. PRENTICE, W.E. Modalidades Terapêuticas em Medicina Esportiva. Ed. Manole. $1^{a}$ Ed. São Paulo, 2002.

20. EMILIANI, Junior; W.R.; TANAKA, C. Postura, flexibilidade da coluna e capacidade funcional em pacientes portadores de lombalgia crônica-Avaliação. Revista de Fisioterapia da Universidade de São Paulo, São Paulo, jul./dez. 2002; 9 (2): 85

21. MEIRELLES, ES. Lombalgia: como diagnosticar e tratar. Revista Brasileira de Medicina, 2003; 60(15): 111-119

22. ZILLI, C.M. Manual de Cinesioterapia /Ginástica Laboral, 2002.

23. GARCIA, N.A.; GONDO, F.L.B.; COSTA, R.A.; CYRILLO, F.N.; COSTA, L.O.P. Efeitos de duas intervenções fisioterapêuticas em pacientes com dor lombar crônica não-específica: viabilidade de um estudo controlado aleatorizado. Revista Brasileira de Fisioterapia, 2011; 15(5): 420-427

24. BIENFIAT, M. Estudo e tratamento do esqueleto fibroso: Fáscia e Pompagens, Editora Summus. $3^{a}$ Ed. São Paulo, 1999
25. SACCO, I.C.N. Método pilates em revista: aspectos biomecânicos de movimentos específicos para reestruturação postural- Estudos de caso. Rev.bras. Ci. E Mov. 2005; 13 (4): 65-78

26. MORENO, M.A. Efeito de um programa de alongamento muscular pelo método de Reeducação Postural Global sobre a força muscular respiratória e a mobilidade tóracoabdominal de homens jovens sedentários. Revista Brasileira de Pneumologia, 2007; 33 (6): 679-686.

27. NEGRELLI, W.F. Hérnia Discal: Procedimentos de tratamento. Acta Ortop Bras, out/dez, 2001; 9(4): 39-45

28. SANTOS, M. Hérnia de Disco: uma revisão clínica, fisiológica e preventiva. Revista Digital, out 2003; 9(65): 1

29. HEINECK, F. Programa de terapia manual para pacientes portadores de hérnia de disco lombar. 2004

30. SACCO, I.C.N.; ANDRADE, M.S.; SOUZA, O.S.; NISIYAMA, M.; CANTUÁRIA,A.L.; MAEDA, F.Y.I.; PIKEL, M. Método pilates em revista: aspectos biomecânicos de movimentos específicos para reestruturação postural - Estudos de caso. Rev. bras.Ci e Mov. 2005; 13(4): 65-78

31. BASILIO, D.R.; PARRA, M.C.G. Os benefícios do pilates na reabilitação de hérnia de disco lombar, 2005

32. MACEDO, C.S.G.; BRIGANO, J.U. Terapia manual e cinesioterapia na dor, incapacidade e qualidade de vida de indivíduos com lombalgia. Revista Espaço para a Saúde, jun. 2009; 10(2): 1-6

33.FOLHADELA, N.N.; MEIJA, D.P.M. Utilização da técnica reeducação postural global (RPG) no Tratamento da lombalgia, 2009

34. BRIGANO, J.U.; MACEDO, C.S.G. Análise da mobilidade lombar e influência da terapia manual e cinesioterapia na lombalgia, jul./dez. 2005;26 ( 2): 75-82

35. MONTEIRO, R.R.; RANGEL, P.M. Efeito das pompagens no tratamento de hérnia discal lombar, 2010

36.TEODORI, R.M.; NEGRI, J.R.; CRUZ, M.C.; MARQUES, A.P. O efeito da Reeducação Postural Global. Rev. bras. fisioter. Mai./jun. 2011; 15(3): 1

37. TENORIO, M.Y.L.C.; VIEIRA, L.C.R. Aspectos associados à lombalgia. Revista Digital out.2012; 17(173): 349-355

38. COSTA, L.M.R.; ROTH, A.; NORONHA, M. O método pilates no Brasil, 2012; 41 (3): 87-92 